\title{
Quincke's Pulse
}

\author{
Mohinder R. Vindhyal, M.D. ${ }^{1}$, Shravani Vindhyal, M.D. ${ }^{1}$, Venkata Subbarao Boppana, M.D. ${ }^{1,2}$ \\ ${ }^{1}$ University of Kansas School of Medicine-Wichita, \\ Department of Internal Medicine \\ ${ }^{2}$ Heartland Cardiology, LLC, Wichita, KS \\ Received October 31, 2018; Accepted for publication December 21, 2018; Published online May 15, 2019
}

A 43-year-old woman with active IV heroin abuse presented with right buttock pain for three days. She had a history of recent aortic valve endocarditis from Streptococcus Milleri which was treated with six weeks of antibiotics. Her examination revealed a temperature of $39.1^{\circ}$ Celsius and blood pressure of 143/45 mmHg. Cardiac auscultation revealed III/IV diastolic murmur and III/VI systolic murmurs over the base of the heart. Upon applying gentle pressure to the nail tip, alternate filling and blanching (Figure l; video files may be accessed at www. kjm.kumc.edu) of the capillary bed concomitant with the cardiac cycle was seen. This is known as Quincke's pulse which is suggestive of severe aortic valve insufficiency.

Transthoracic echocardiography confirmed severe aortic regurgitation secondary to aortic valve endocarditis. The patient was found to have septic arthritis of right sacroiliac joint and right piriformis muscle abscess, treated with pelvic drainage. Blood cultures from the admission grew Streptococcus intermedius. The patient was treated with intravenous antibiotics and had a surgical aortic valve replacement. The patient remained symptom free and did well afterwards.
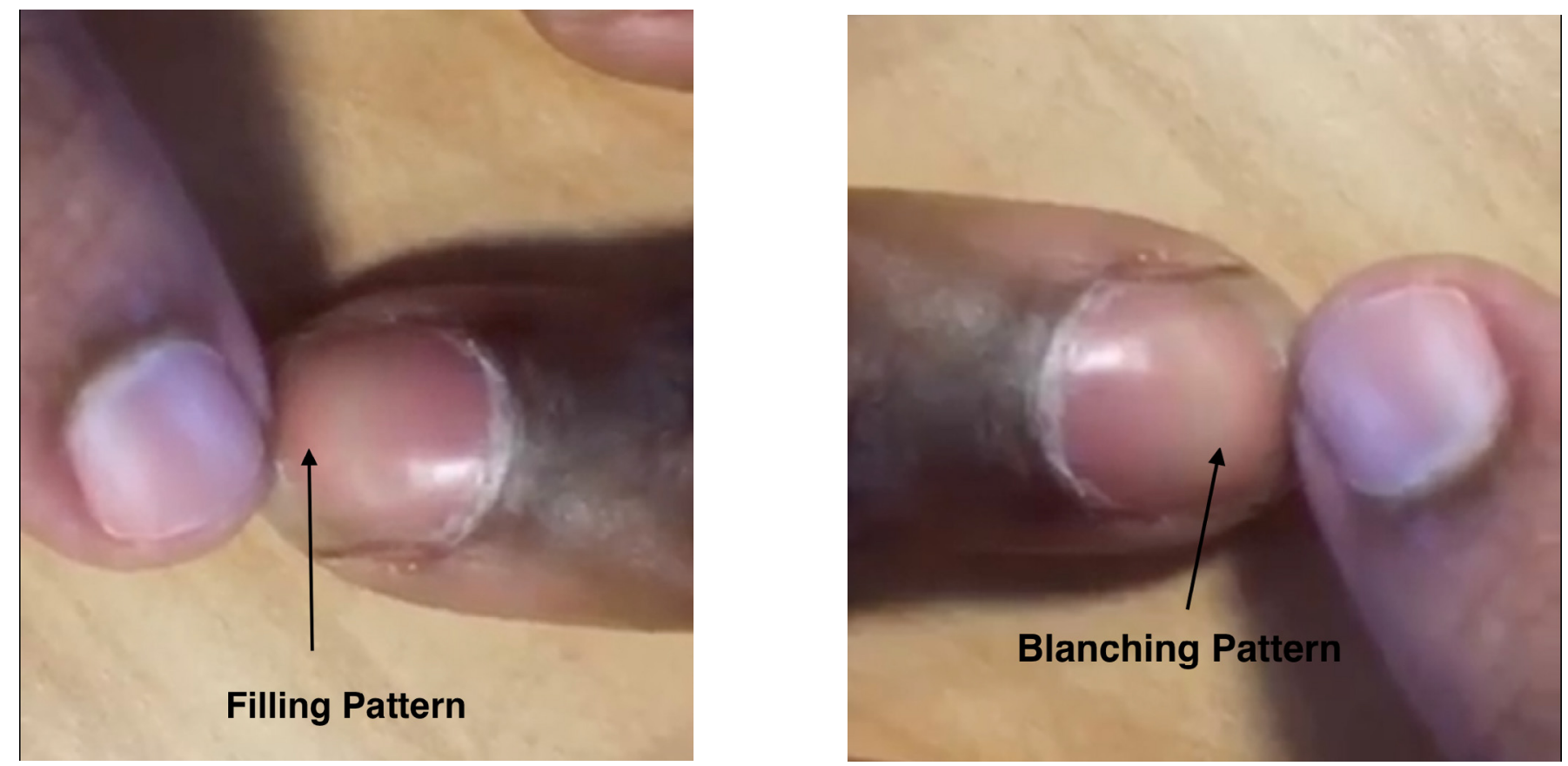

Figure l. The filling (left) and blanching pattern (right) of the Quincke's pulse.

\section{DISCUSSION}

Quincke's pulse is a clinical sign of severe aortic valve insufficiency where there is repeated blushing and blanching of nail capillaries. ${ }^{1,2}$ Aortic insufficiency causes the regurgitant blood to flow into the left ventricle causing increased systolic stroke volume and simultaneous lowering of diastolic pressure leading to widened pulse pressure, resulting in the capillary pulsation.

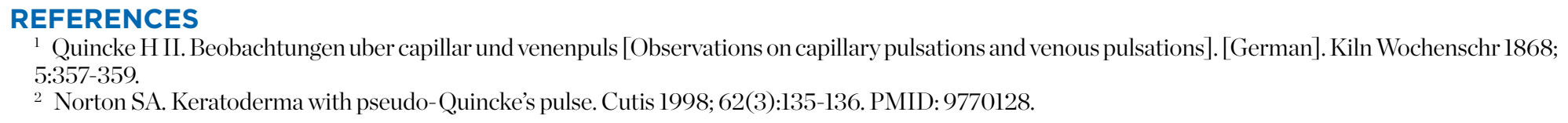

${ }^{1}$ Quincke H II. Beobachtungen uber capillar und venenpuls [Observations on capillary pulsations and venous pulsations]. [German]. Kiln Wochenschr 1868; 5:357-359.

${ }^{2}$ Norton SA. Keratoderma with pseudo-Quincke’s pulse. Cutis 1998; 62(3):135-136. PMID: 9770128. 